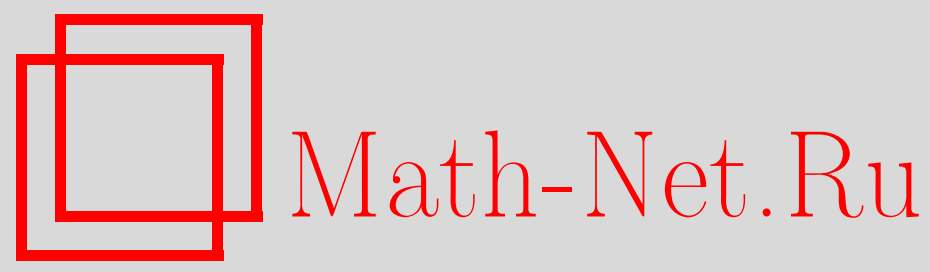

В. И. Буренков, Л. Д. Кудрявцев, И. В. Неверов, Об одном тождестве для разностей произвольного порядка, Матем. заметки, 1998, том 64, выпуск 2, 302-307

DOI: https://doi.org/10.4213/mzm1398

Использование Общероссийского математического портала Math-Net.Ru подразумевает, что вы прочитали и согласны с пользовательским соглашением http://www . mathnet.ru/rus/agreement

Параметры загрузки:

IP: 52.205 .19 .152

26 апреля 2023 г., 10:10:10

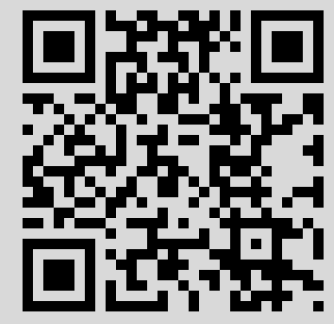




\section{ОБ ОДНОМ ТОЖДЕСТВЕ ДЛЯ РАЗНОСТЕЙ ПРОИЗВОЛЬНОГО ПОРЯДКА}

\section{В.И. Буренков, Л. Д. Кудрявцев, И. В. Неверов}

Докажем равенство

$$
\sum_{i=0}^{2 m-1} a_{i m} b_{i}=\sum_{k=0}^{m-1} \lambda_{k m} \Delta^{m} b_{k}, \quad m \in \mathbb{N},
$$

в котором числа $b_{k}, k \in \mathbb{Z}$, произвольны, а числа $a_{i m}$ и $\lambda_{k m}$ заданы следуюшим образом:

$$
a_{i m}=\sum_{j=i+1}^{m} \frac{(-1)^{j+1}}{j} C_{m}^{j}+\sum_{\substack{(i+1) / 2 \leqslant j \leqslant i \\ 1 \leqslant j \leqslant m}} \frac{(-1)^{j}}{j} C_{m}^{j}, \quad i=\overline{0,2 m-1}
$$

(если нижний предел оказьвается больше верхнего, то считаем сумму равной 0),

$$
\lambda_{k m}=(-1)^{m} a_{k m}+(-1)^{k+1} \sum_{j=0}^{k-1}(-1)^{j} C_{m}^{k-j} \lambda_{j m}, \quad k=\overline{0, m-1},
$$

a $\Delta^{m} b_{k}-$ разность порядка $m$ :

$$
\Delta^{m} b_{k}=\sum_{j=0}^{m}(-1)^{m+j} C_{m}^{j} b_{k+j}
$$

где под $b_{k}$ можно понимать не только числа, но и элементы любого линейного векторного пространства.

Равенство (1) применяется при доказательстве существования разложения локально суммируемых функций в ряды по интегралам от разностей высших порядков рассматриваемых функций [1].

Доказательство тождества (1) основано на двух теоремах.

Теорема 1. Даны числа $a_{0}, a_{1}, \ldots, a_{2 m-1}, m \in \mathbb{N}$. Следующие два условия эквивалентнь:

$$
\begin{gathered}
\exists \mu_{0}, \mu_{1}, \ldots, \mu_{m-1} \quad \forall b_{0}, b_{1}, \ldots, b_{2 m-1} \quad \sum_{i=0}^{2 m-1} a_{i} b_{i}=\sum_{k=0}^{m-1} \mu_{k} \Delta^{m} b_{k}, \\
\sum_{i=0}^{2 m-1} a_{i} i^{l}=0, \quad l=\overline{0, m-1} .
\end{gathered}
$$

ЗАмечАниЕ 1. Условие (4) можно переписать в виде

$$
\exists \mu_{0}, \mu_{1}, \ldots, \mu_{m-1} \quad \sum_{i=1}^{2 m} a_{i-1} e_{i}=\sum_{k=1}^{m} \mu_{k-1} \Delta^{m} e_{k}
$$

где $\boldsymbol{e}_{k}-k$-й вектор ортонормированного базиса в $\mathbb{R}^{2 m}$. Так как $\left\{\Delta^{m} \boldsymbol{e}_{k}\right\}_{k=1}^{m}$ - линейно независимая система векторов, числа $\mu_{i}$ определяются однозначно.

Работа выполнена при финансовой поддержке программы "Ведущие научные школы", грант № 96-15-96102. 
ДокАЗАТЕльство. (4) $\Longrightarrow(5)$. Возьмем $b_{i}=i^{l}, l=\overline{0, m-1}$. Тогда из (4) вытекает

$$
\sum_{i=1}^{2 m-1} a_{i} i^{l}=\sum_{i=1}^{m-1} \mu_{i} \Delta^{m} i^{l}=0
$$

так как при $l=\overline{0, m-1}$ имеет место равенство $\Delta^{m} i^{l}=0$, т.е.

$$
\sum_{i=0}^{m}(-1)^{m+i} i^{l} C_{m}^{i}=0, \quad l=\overline{0, m-1} .
$$

Это следует из того, что для любых $h \in \mathbb{R}$ и $x>0$ при $l=\overline{0, m-1}$ имеет место $\Delta_{h}^{m} x^{l}=0$.

$(5) \Longrightarrow(4)$. Положим $\widetilde{a}_{i}=a_{i}, \widetilde{b}_{i}=b_{i}, i=\overline{0,2 m-1} ; \widetilde{a}_{i}=\widetilde{b}_{i}=0, i \geqslant 2 m$. Тогда

$$
\sum_{i=1}^{2 m-1} a_{i} b_{i}=\sum_{i=1}^{\infty} \widetilde{a}_{i} \widetilde{b}_{i}=-\sum_{i=0}^{\infty}\left(\sum_{j=0}^{i} \widetilde{a}_{j}\right) \Delta \widetilde{b}_{i} .
$$

Действительно, положим $\eta_{i}=\sum_{k=0}^{i-1} \widetilde{a}_{k}$, так что $\eta_{1}=\widetilde{a}_{0}, \Delta \eta_{i}=\widetilde{a}_{i}$; имеем

$$
\begin{gathered}
\Delta(\eta \widetilde{b})_{i}=\eta_{i+1} \Delta \widetilde{b}_{i}+\widetilde{b}_{i} \Delta \eta_{i}=\eta_{i+1} \Delta \widetilde{b}_{i}+\widetilde{b}_{i} \widetilde{a}_{i}, \quad \sum_{i=1}^{\infty} \Delta(\eta \widetilde{b})_{i}=-\eta_{1} \widetilde{b}_{1}=\sum_{i=1}^{\infty}\left(\eta_{i+1} \Delta \widetilde{b}_{i}+\widetilde{b}_{i} \widetilde{a}_{i}\right), \\
-\widetilde{a}_{0} \widetilde{b}_{1}=\sum_{i=1}^{\infty}\left(\eta_{i+1} \Delta \widetilde{b}_{i}+\widetilde{b}_{i} \widetilde{a}_{i}\right), \quad \widetilde{a}_{0} \widetilde{b}_{0}-\widetilde{a}_{0} \widetilde{b}_{1}=\sum_{i=0}^{\infty} \widetilde{a}_{i} \widetilde{b}_{i}+\sum_{i=1}^{\infty} \eta_{i+1} \Delta \widetilde{b}_{i}, \\
0=\sum_{i=0}^{\infty} \widetilde{a}_{i} \widetilde{b}_{i}+\widetilde{a}_{0}\left(\widetilde{b}_{1}-\widetilde{b}_{0}\right)+\sum_{i=1}^{\infty} \eta_{i+1} \Delta \widetilde{b}_{i}=\sum_{i=0}^{\infty} \widetilde{a}_{i} \widetilde{b}_{i}+\sum_{i=0}^{\infty} \eta_{i+1} \Delta \widetilde{b}_{i},
\end{gathered}
$$

откуда

$$
\sum_{i=0}^{\infty} \widetilde{a}_{i} \widetilde{b}_{i}=-\sum_{i=0}^{\infty} \eta_{i+1} \Delta \widetilde{b}_{i}
$$

что и требовалось доказать.

Применив это преобразование $m-1$ раз, получим

$$
\sum_{i=0}^{\infty} \widetilde{a}_{i} \widetilde{b}_{i}=\sum_{i=0}^{\infty} \mu_{i} \Delta^{m} \widetilde{b}_{i}
$$

где

$$
\mu_{i}=(-1)^{m} \sum_{j_{m}=0}^{i} \sum_{j_{m-1}=0}^{j_{m}} \cdots \sum_{j_{1}=0}^{j_{2}} \widetilde{a}_{j_{1}}=\sum_{j=0}^{i} C_{i-l+m-1}^{m-1} \widetilde{a}_{j}, \quad i \in \mathbb{Z}^{+} .
$$

Последнее равенство доказьвается следуюшим образом. Рассмотрим $j$-й элемент $\tilde{a}_{j}$. Правее него $(i+1)-(j+1)=i-j$ элементов и на 1 больше, т.е. $i-j+1$, позиций для отметок, соответствуюших верхним границам сумм. Коэффицциент в сумме

$$
\sum_{j_{m}=0}^{i} \cdots \sum_{j_{1}=0}^{j_{2}} \tilde{a}_{j_{1}}
$$

при $\widetilde{a}_{j}$ равен числу способов разместить $m-1$ отметок (так как одна из $m$ отметок фиксированадля внешней суммы) на $i-j+1$ позициях при сохранении порядка отметок, причем отметки могут накладьваться. Каждую $i$-ю отметку $(i=\overline{2, m-1})$ сдвинем на $+(i-1)$ относительно выбранного для нее места. Тогда добавится $m-2$ новых позиций и наше множество выборок (или размещений) 
взаимно однозначно отобразится на множество неупорядоченных выборок из $i-j+1+m-2$ по $m-1$. Таким образом, коэффициент при $\widetilde{a}_{j}$ равен $C_{i-j+m-1}^{m-1}$. вид

Поскольку при $i \geqslant 2 m \Delta^{m} \widetilde{b}_{i}=0$, а при $i \leqslant m-1 \Delta^{m} \widetilde{b}_{i}=\Delta^{m} b_{i}$, равенство (7) принимает

$$
\sum_{i=0}^{2 m-1} a_{i} b_{i}=\sum_{i=0}^{m-1} \mu_{i} \Delta^{m} b_{i}+\sum_{i=m}^{2 m-1} \mu_{i} \Delta^{m} \widetilde{b}_{i} .
$$

Поскольку $C_{k}^{m-1}=C_{k+1}^{m-1}-C_{k}^{m-2}$, при $m \geqslant 2$ получаем

$$
\begin{aligned}
\sum_{j=0}^{i} C_{i-j+m-1}^{m-1} a_{j} & =\sum_{j=0}^{i} C_{i+1-j+m-1}^{m-1} a_{j}-\sum_{j=0}^{i} C_{i+1-j+m-2}^{m-2} a_{j} \\
& =\sum_{j=0}^{i+1} C_{i+1-j+m-1}^{m-1} a_{j}-\sum_{j=0}^{i+1} C_{i+1-j+m-2}^{m-2} a_{j}
\end{aligned}
$$

Повторяя эту процедуру, приходим к тому, что при $m \geqslant s+1$

$$
\sum_{j=0}^{i} C_{i-j+m-1}^{m-1} a_{j}=\sum_{k=0}^{s}(-1)^{k} C_{s}^{k} \sum_{j=0}^{i+s} C_{i+s-j+m-1-k}^{m-1-k} a_{j}
$$

При $m=1$ исходная сумма уже имеет требуемый вид, что соответствует случаю $s=0$. Выбирая здесь $i+s=2 m-1, i=\overline{m, 2 m-1}$, получаем

$$
\sum_{j=0}^{i} C_{i-j+m-1}^{m-1} a_{j}=\sum_{k=0}^{2 m-1-i}(-1)^{k} C_{2 m-1-i}^{k} \sum_{j=0}^{2 m-1} C_{2 m-1-j+m-1-k}^{m-1-k} a_{j}, \quad k=\overline{0, m-1} .
$$

Поскольку $C_{2 m-1-j+m-1-k}^{m-1-k}$ многочлен степени $m-1-k$ от $j$, в силу (5) имеем

$$
\sum_{j=0}^{2 m-1} C_{2 m-1-j+m-1-k}^{m-1-k} a_{j}=0
$$

Следовательно,

$$
\sum_{j=0}^{i} C_{i-j+m-1}^{m-1} a_{j}=0, \quad i=\overline{m, 2 m-1}
$$

значит $\mu_{i}=0, i=\overline{m, 2 m-1}$, и (8) переходит в (4).

ЗАмЕч АниЕ 2 . При выполнении условия (4) числа $\mu_{i}, i=\overline{0, m-1}$, определяются однозначно:

$$
\mu_{i}=(-1)^{m} \sum_{j=0}^{i} C_{i-j+m-1}^{m-1} a_{j}
$$

Рассмотрим ньютоновские степени $(k)_{l}=k(k+1) \cdots(k+l-1), k \in \mathbb{Z}^{+}, l \in \mathbb{N}$; считаем $(k)_{0}=1$ (в том числе $\left.(0)_{0}=1\right)$. Отметим, что

$$
(k)_{l}=k(k+1)_{l-1}, \quad \sum_{k=0}^{i}(k)_{l}=\frac{(i)_{l+1}}{l+1}, \quad l \in \mathbb{N} .
$$

Мы будем пользоваться эквивалентным (6) равенством

$$
\sum_{j=0}^{m}(-1)^{j}(j)_{l} C_{m}^{j}=0, \quad l=\overline{0, m-1}
$$


Кроме того, рассмотрим усредненные ньютоновские степени

$$
\overline{(k)}_{l}=\frac{1}{k} \sum_{i=k}^{2 k-1}(i)_{l}, \quad k \in \mathbb{N}, \quad l \in \mathbb{Z}^{+}
$$

считаем $\overline{(0)}_{l}=0$ при $l \in \mathbb{N}$ и $\overline{(0)}_{0}=1$. Отметим, что согласно (9) при $k, l \in \mathbb{N}$

$$
\begin{aligned}
\overline{(k)}_{l} & =\frac{1}{k}\left(\sum_{i=0}^{2 k-1}(i)_{l}-\sum_{i=0}^{k-1}(i)_{l}\right)=\frac{1}{k(l+1)}\left((2 k-1)_{l+1}-(k-1)_{l+1}\right) \\
& =\frac{1}{l+1}\left(2(2 k-1)(2 k+1)_{l-1}-(k-1)(k+1)_{l-1}\right)=P_{l}(k)
\end{aligned}
$$

- многочлен степени $l$. Согласно (6)

$$
\sum_{j=0}^{m}(-1)^{j} P_{l}(j) C_{m}^{j}=0, \quad l<m .
$$

Однако,

$$
\begin{gathered}
P_{l}(0)=\frac{1}{l+1}\left(-2(1)_{l-1}+(1)_{l-1}\right)=-\frac{(1)_{l-1}}{l+1} \neq \overline{(0)}_{l}=0, \quad l \geqslant 1 \\
\sum_{j=0}^{m}(-1)^{j} \overline{(j)}_{l} C_{m}^{j}=\sum_{j=0}^{m}(-1)^{j}\left(\overline{(j)}_{l}-P_{l}(j)\right) C_{m}^{j}=(-1)^{0}\left(\overline{(0)_{l}}-P_{l}(0)\right) C_{m}^{0}=-P_{l}(0)=\frac{(1)_{l-1}}{l+1} .
\end{gathered}
$$

Таким образом,

$$
\sum_{j=0}^{m}(-1)^{j} \overline{(j)_{l}} C_{m}^{j}=\frac{(1)_{l-1}}{l+1}, \quad l=\overline{1, m-1} .
$$

ТЕОРЕма 2. Числа (2) удовлетворяют равенствам

$$
\sum_{i=0}^{2 m-1}(i)_{l} a_{i m}=0, \quad l=\overline{0, m-1}
$$

что эквивалентно условию (5).

ДоКАЗАТЕльСтво. Из (8) следует, что

$$
\begin{gathered}
\sum_{i=0}^{2 m-1}(i)_{l} a_{i m}=\sum_{i=0}^{m-1}(i)_{l} \sum_{j=i+1}^{m} \frac{(-1)^{j+1}}{j} C_{m}^{j}+\sum_{i=1}^{m-1}(i)_{l} \sum_{(i+1) / 2 \leqslant j \leqslant i} \frac{(-1)^{j}}{j} C_{m}^{j} \\
+\sum_{i=m}^{2 m-1}(i)_{l} \sum_{(i+1) / 2 \leqslant j \leqslant m} \frac{(-1)^{j}}{j} C_{m}^{j}=\Sigma_{1}+\Sigma_{2}+\Sigma_{3}, \\
\Sigma_{2}=\sum_{j=1}^{m-1}\left(\sum_{j \leqslant i \leqslant \min \{m-1,2 j-1\}}(i)_{l}\right) \frac{(-1)^{j}}{j} C_{m}^{j} \\
=\sum_{1 \leqslant j \leqslant m / 2}\left(\sum_{i=j}^{2 j-1}(i)_{l}\right) \frac{(-1)^{j}}{j} C_{m}^{j}+\sum_{\Sigma_{3}=} \sum_{(m+1) / 2 \leqslant j \leqslant m}\left(\sum_{i=m}^{m-1}(i)_{l}\right) \frac{(-1)^{j}}{j} C_{m}^{j}, \\
\left(\sum_{i=j}^{2 j-1}(i)_{l}\right) \frac{(-1)^{j}}{j} C_{m}^{j},
\end{gathered}
$$




$$
\begin{aligned}
\Sigma_{2}+\Sigma_{3}= & \sum_{1 \leqslant j \leqslant m / 2} \overline{(j)}_{l}(-1)^{j} C_{m}^{j}+\sum_{m / 2<j \leqslant m-1}\left(\sum_{i=j}^{m-1}(i)_{l}\right) \frac{(-1)^{j}}{j} C_{m}^{j} \\
& +\sum_{(m+1) / 2 \leqslant j \leqslant m}\left(\sum_{i=m}^{2 j-1}(i)_{l}\right) \frac{(-1)^{j}}{j} C_{m}^{j} .
\end{aligned}
$$

При $m=2 k$

$$
\begin{aligned}
\Sigma_{2}+\Sigma_{3} & =\sum_{j=1}^{k} \overline{(j)}_{l}(-1)^{j} C_{2 k}^{j}+\sum_{j=k+1}^{2 k-1}\left(\frac{1}{j} \sum_{i=j}^{2 k-1}(i)_{l}\right)(-1)^{j} C_{2 k}^{j}+\sum_{j=k+1}^{2 k}\left(\frac{1}{j} \sum_{i=2 k}^{2 j-1}(i)_{l}\right)(-1)^{j} C_{2 k}^{j} \\
& =\sum_{j=1}^{k} \overline{(j)}_{l}(-1)^{j} C_{2 k}^{j}+\sum_{j=k+1}^{2 k-1} \overline{(j)}_{l}(-1)^{j} C_{2 k}^{j}+\overline{(2 k)}_{l}=\sum_{j=1}^{2 k} \overline{(j)}_{l}(-1)^{j} C_{2 k}^{j} .
\end{aligned}
$$

При $m=2 k+1$

$$
\begin{aligned}
\Sigma_{2}+\Sigma_{3} & =\sum_{j=1}^{k} \overline{(j)}_{l}(-1)^{j} C_{m}^{j}+\sum_{j=k+1}^{2 k}\left(\frac{1}{j} \sum_{i=j}^{2 k}(i)_{l}\right)(-1)^{j} C_{m}^{j}+\sum_{j=k+1}^{2 k+1}\left(\frac{1}{j} \sum_{i=2 k+1}^{2 j-1}(i)_{l}\right)(-1)^{j} C_{m}^{j} \\
& =\sum_{j=1}^{k} \overline{(j)}_{l}(-1)^{j} C_{m}^{j}+\sum_{j=k+1}^{2 k} \overline{(j)}_{l}(-1)^{j} C_{2 k}^{j}+\overline{(2 k+1)}_{l}(-1)^{2 k+1} C_{m}^{2 k+1} \\
& =\sum_{j=1}^{2 k+1} \overline{(j)}_{l}(-1)^{j} C_{m}^{j} .
\end{aligned}
$$

Таким образом,

$$
\Sigma_{2}+\Sigma_{3}=\sum_{j=1}^{m} \overline{(j)}_{l}(-1)^{j} C_{m}^{j}
$$

Далее,

$$
\Sigma_{1}=\sum_{j=1}^{m}\left(\sum_{i=0}^{j-1}(i)_{l}\right) \frac{(-1)^{j+1}}{j} C_{m}^{j}
$$

откуда

$$
\sum_{i=0}^{2 m-1}(i)_{l} a_{i m}=\sum_{j=1}^{m}\left(\sum_{i=0}^{j-1}(i)_{l}\right) \frac{(-1)^{j+1}}{j} C_{m}^{j}+\sum_{j=1}^{m} \overline{(j)}_{l}(-1)^{j} C_{m}^{j}
$$

При $l=0$

$$
\sum_{i=0}^{j-1}(i)_{l}=j \Longrightarrow \Sigma_{1}=\sum_{j=1}^{m}(-1)^{j+1} C_{m}^{j}, \quad \Sigma_{2}+\Sigma_{3}=\sum_{j=1}^{m}(-1)^{j} C_{m}^{j} \Longrightarrow \sum_{i=0}^{2 m-1}(i)_{l} a_{i}=0
$$

При $l>0$ согласно (9)

$$
\sum_{i=0}^{j-1}(i)_{l}=\frac{(j-1)_{l+1}}{l+1}
$$


откуда согласно (11) и (10)

$$
\begin{aligned}
\sum_{i=0}^{2 m-1}(i)_{l} a_{i} & =\frac{1}{l+1} \sum_{j=1}^{m} \frac{(j-1)_{l+1}}{j}(-1)^{j+1} C_{m}^{j}+\sum_{j=1}^{m} \overline{(j)}_{l}(-1)^{j} C_{m}^{j} \\
& =\frac{1}{l+1} \sum_{j=1}^{m}(j-1)(j+1)_{l-1}(-1)^{j+1} C_{m}^{j}+\frac{(1)_{l-1}}{l+1} \\
& =\frac{1}{l+1} \sum_{j=0}^{m}(j-1)(j+1)_{l-1}(-1)^{j+1} C_{m}^{j}=\sum_{j=0}^{m}(-1)^{j} Q_{l}(j) C_{m}^{j}=0
\end{aligned}
$$

Комбинируя теоремы 1 и 2 , получаем равенство (1), где числа $\lambda_{k m}$ согласно замечанию 2 определены однозначно. Равенство (3) есть следствие равенства (1). Действительно, перегруппировывая слагаемые в правой части (1), получаем

$$
\sum_{i=0}^{2 m-1} a_{i m} b_{i}=\sum_{k=0}^{2 m-1} b_{k}\left(\sum_{j=0}^{k} \lambda_{j m}(-1)^{m+k-j} C_{m}^{k-j}\right)
$$

значит

$$
(-1)^{m} a_{k m}=\sum_{j=0}^{k} \lambda_{j m}(-1)^{k-j} C_{m}^{k-j}=\lambda_{k m}+\sum_{j=0}^{k-1}(-1)^{k+j} C_{m}^{k-j} \lambda_{j m}, \quad k=\overline{0,2 m-1}
$$

откуда следует (3).

\section{СПИСОК ЦИТИРОВАННОЙ ЛИТЕРАТУРЫ}

1. Кудрявцев Л. Д. // Тр. МИАН. 1995. Т. 210. С. 129-170. 